\title{
PCE Regimen
}

National Cancer Institute

\section{Source}

National Cancer Institute. PCE Regimen. NCI Thesaurus. Code C9704.

A chemotherapy regimen consisting of cisplatin, cyclophosphamide, and vindesine that

may be used in the treatment of non-small cell lung cancer (NSCLC). 